\title{
3-HYDROXYBUTYRATE CO-INFUSED WITH NORADRENALINE DECREASES RESULTING PLASMA LEVELS OF NORADRENALINE IN WISTAR RATS
}

\author{
X. CAÑAS ${ }^{1}$, D. SANCHIS ${ }^{1}$, G. GÓMEZ ${ }^{1}$, J. M. CASANOVAS ${ }^{2}$, F. ARTIGAS ${ }^{2}$, J. A. FERNÁNDEZ-LÓPEZ ${ }^{1}$, \\ X. REMESAR ${ }^{1}$ AND M. ALEMANY ${ }^{1, *}$ \\ ${ }^{1}$ Departament de Bioquímica i Biologia Molecular, Facultat de Biologia, Universitat de Barcelona, \\ Avinguda Diagonal 645, 08028 Barcelona, Spain and 2Institut d'Investigacions Biomèdiques de Barcelona, \\ Barcelona, Spain
}

Accepted 28 July 1997

\begin{abstract}
Summary
Pentobarbital-anaesthetized male Wistar rats were infused with $6 \mu \mathrm{g} \mathrm{kg}^{-1} \mathrm{~min}^{-1}$ of noradrenaline. The infusion was supplemented with $8.5 \mathrm{mg} \mathrm{kg}^{-1} \mathrm{~min}^{-1}$ of $\mathrm{D}$-3hydroxybutyrate (3-OHB) for $15 \mathrm{~min}$ in order to determine its effect on the adrenergic response of the rat. Plasma levels of noradrenaline rose to a plateau of approximately $50 \mathrm{nmoll}^{-1}$ with infusion. In the group infused with noradrenaline alone, noradrenaline levels were maintained for $1 \mathrm{~h}$. Supplementation with 3-OHB induced a decrease in plasma noradrenaline level that was inversely correlated with 3-OHB level. Aortic and interscapular brown adipose

temperature in either tissue. Blood 3-OHB concentration increased to $1.2 \mathrm{mmoll}^{-1}$ during 3-OHB infusion, decreasing rapidly at the end of infusion. Blood glucose levels increased with noradrenaline infusion; the presence of high 3-OHB levels decreased glucose concentration. The effects observed were transient and dependent on 3-OHB concentration; these effects may help explain most of the other effects of noradrenaline described here. The role of 3-OHB as a regulator of adrenergic responses seems to be part of a complex fail-safe mechanism which prevents wasting.
\end{abstract} tissue temperatures increased with noradrenaline infusion, but the rise was arrested by 3-OHB; replacing 3-OHB with glucose had no effect. Infusion of saline, glucose or 3-OHB in the absence of noradrenaline did not induce a rise in
Key words: 3-hydroxybutyrate, ketone bodies, thermogenesis, noradrenaline, brown adipose tissue, body temperature, rat.

\section{Introduction}

Adaptive thermogenesis by brown adipose tissue (BAT) is directly related to noradrenergic stimulation (Foster and Frydman, 1978), both under cold-exposure (Foster and Frydman, 1979) or when excess energy is available (Rothwell and Stock, 1979). Continuous infusion of rats with catecholamines results in increased heat production and a rise in blood temperature (Hugie et al. 1992; Zylan and Carlisle, 1992) as a consequence of the stimulation of BAT (Trayhurn and Ashwell, 1987). The effects of noradrenaline infusion include increases in circulating glucose levels (Nikami et al. 1992), basically a consequence of massive glycogen breakdown (Shiota et al. 1992), as well as marked changes in blood flow distribution and cardiac output (Gordon, 1990).

Ketone bodies are synthesized by the liver under conditions of scarce glucose combined with marked lipolysis and the consequent availability of fatty acids (Ubukata et al. 1992). Ketone bodies are produced during starvation (Balasse, 1979; Balasse and Féry, 1989), diabetes (Sherwin et al. 1976; Féry and Balasse, 1985; Balasse and Féry, 1989) or the consumption of fatty low-carbohydrate (ketogenic) diets (Ide et al. 1984).
The large change in blood levels of 3-hydroxybutyrate (3OHB) and acetoacetate from the basal to the ketotic condition means that these levels may be a signal to determine which substrates are oxidized (Hetenyi and Ferraroto, 1985; Ohmori et al. 1990; Metcalfe et al. 1992). It has been found that 3OHB acts as a signal that limits BAT substrate oxidation and thermogenesis (Pokasanieva et al. 1993), thus preventing unwanted wastage of substrates under conditions of starvation or altered substrate utilization (De Pergola et al. 1990). This effect occurs through modulation of the response of BAT (Pokasanieva et al. 1993), but also by a direct interaction between 3-OHB and adrenergic receptors (De Pergola et al. 1990). The results presented here show the direct effects of 3OHB on anaesthetized rats constantly infused with noradrenaline.

\section{Materials and methods}

Two-month-old male Wistar rats (from Charles River, France) weighing $240-260 \mathrm{~g}$ were used. The animals were kept

\footnotetext{
*Author for correspondence (e-mail: alemany@ porthos.bio.ub.es).
} 


\section{X. CAÑAS AND OTHERS}

under controlled conditions of feeding (B\&K, Sant Vicent dels Horts, Spain), illumination (light on from 08:00 to 20:00 h), temperature $\left(21-22^{\circ} \mathrm{C}\right)$ and humidity $(70 \%)$ in individual polypropylene-bottomed cages until the experiments were performed. All experiments took place between 08:00 and 10:00 h. The rats were anaesthetized with an intraperitoneal injection of $50 \mathrm{mg} \mathrm{kg}^{-1}$ sodium pentobarbital (Sigma, St Louis, $\mathrm{MO}, \mathrm{USA}$ ) in $9 \mathrm{gl}^{-1} \mathrm{NaCl}$. The rats were then placed in a chamber with the temperature controlled at $28-30^{\circ} \mathrm{C}$. Two Ktype thermocouple temperature probes (Kane May, Welwyn Garden City, Herts, UK) were inserted, one on the lower aorta wall and the other between the interscapular BAT masses, as described previously (Closa et al. 1993). The probes were connected to a Data logger (type KM-1420, from Kane May) which recorded temperatures at $10 \mathrm{~s}$ intervals. These data were converted into $3 \mathrm{~min}$ means in order to allow comparisons between series of rats.

The lower vena cava was then cannulated using P10 polyethylene catheter tubing (Clay Adams, Parsipanny, NJ, USA) for continuous infusion. A second cannula was also placed on the lower vena cava, more distal with respect to the heart, and filled with heparinized saline to keep it functional for sampling.

The infusion apparatus consisted of a slow infusion syringe pump (Precidor 5003, Infors AG, Bottmingen, Germany) filled with saline; the saline was injected at a rate of $4 \mathrm{ml} \mathrm{h}^{-1} \mathrm{~kg}^{-1}$. The syringe efflux was distributed by a $\mathrm{Y}$-piece into two containers filled with $280 \mu \mathrm{moll}^{-1} \quad \mathrm{~L}-(+)$-noradrenaline bitartrate (Sigma) in $9 \mathrm{gl}^{-1} \mathrm{NaCl}$; one of these contained, in addition, $1 \mathrm{moll}^{-1} \mathrm{D}-(-)-3$-hydroxybutyric acid sodium salt (Sigma) and $10 \mathrm{mg} \mathrm{l}^{-1}$ Neutral Red (Sigma, used to colour this solution in order to check its flow) in $50 \mathrm{mmoll}^{-1}$ phosphate buffer (pH7.4). The flow of either noradrenaline or noradrenaline +3 -OHB could be selected by means of a threeway tap, allowing a rapid change of infusion fluid into the lower vena cava without a change in the rate of injection or in the pressure inside the system.

We used an infusion fluid buffered to $\mathrm{pH} 7.4$ to minimize the negative effects of the infusion on the acid-base equilibrium of the rat. The noradrenaline solution was prepared freshly for each rat immediately $(5-10 \mathrm{~min})$ before use. Both solutions (noradrenaline alone and supplemented with 3-OHB) were prepared in parallel, i.e. noradrenaline was dissolved in a volume of saline made up with freshly prepared pure water (Milli-Q quality, Millipore, Bedford, MA, USA); a volume of stock 3-OHB (brought to $\mathrm{pH}$ 7.4) and Neutral Red solution or plain pure water was then added. Finally, the concentrated buffer (phosphates, final concentration $50 \mathrm{mmol}^{-1}$ ) was added and mixed. The parallel containers were then completely filled with the twin solutions. At the end of the experiment, one sample of the infusion solution was taken and frozen after adding glutathione and internal standard as for the blood samples. This method was used to determine the degree of preservation of noradrenaline in the samples. There were no significant differences in noradrenaline content between the standards containing 3-OHB and those not containing it. The losses of noradrenaline were quite uniform, 5.1 $\pm 1.7 \%$ (mean \pm S.E.M., $N=10$ ) for freshly taken samples. Storage time affected the noradrenaline content (approximately $8 \%$ was lost over 2 weeks).

The closed system used for storage and delivery of the infusion fluid prevented its contact with oxygen, thus limiting noradrenaline oxidation (West, 1952; Hughes and Smith, 1978). Neither the utilization of a thermoneutral chamber $\left(28-30^{\circ} \mathrm{C}\right.$ ) nor a neutral $\mathrm{pH}$ in the buffered infusion fluid affected the noradrenaline concentration in the infused solution for the duration of the experiment and storage.

Rats were anaesthetized and prepared for infusion in 10-13 min; infusion experiments were then carried out in the heated chamber for a further $60 \mathrm{~min}$.

A group of five rats was infused with $100 \mathrm{mmoll}^{-1}$ glucose instead of noradrenaline, another group of five rats with $1 \mathrm{moll}^{-1} 3$-OHB alone, without noradrenaline; a third group of five rats was infused with noradrenaline as indicated above, but the 3 -OHB was replaced by $100 \mathrm{mmol}^{-1}$ glucose. Finally, a group of five additional control rats was infused with $9 \mathrm{gl}^{-1}$ $\mathrm{NaCl}$ only.

Before the beginning of the infusion procedure, the aortic temperature of the rats was checked. Even in the heated chamber, there was a slow tendency to hypothermia caused by anaesthesia (Sessler, 1991); infusion began when the rat maintained a temperature of $36^{\circ} \mathrm{C}$ for $30 \mathrm{~s}$, thus ensuring an added degree of uniformity in the responses observed.

Blood samples in this series of rats were limited to approximately $0.120 \mathrm{ml}$ (every $6 \mathrm{~min}$ ) in each extraction by drawing the blood through short pieces of capillary tube used as blood containers. Blood samples were blown out of the tubes into small Eppendorf plastic vials; they were centrifuged at $10000 \mathrm{~g}$ for $7 \mathrm{~min}$ to obtain plasma. Samples of $75 \mu \mathrm{l}$ of plasma were mixed with $675 \mu \mathrm{l}$ of water, $50 \mu \mathrm{l}$ of $15 \mathrm{mmol}^{-1}$ reduced glutathione (Sigma), $50 \mu \mathrm{l}$ of $90 \mathrm{mmol}^{-1}$ EDTA (Sigma) and $60 \mu \mathrm{l}$ of $130 \mathrm{nmoll}^{-1}$ dihydroxybenzylamine (Sigma, internal standard). The samples were then shaken (with an orbital mixer for $30 \mathrm{~min}$ at $4^{\circ} \mathrm{C}$ ) with $20 \mathrm{mg}$ of dry alumina (Merck, Darmstadt, Germany) and $400 \mu \mathrm{l}$ of $500 \mathrm{mmoll}^{-1}$ Tris- $\mathrm{HCl}$ buffer, $\mathrm{pH} 8.6$. The samples were centrifuged for $10 \mathrm{~min}$ at $2000 \mathrm{~g}$ at $4^{\circ} \mathrm{C}$. The pellet was resuspended in $1 \mathrm{ml}$ of 3 mmol $^{-1}$ EDTA buffered at $\mathrm{pH} 7.0$ and centrifuged again for $7 \mathrm{~min}$; this washing was repeated three times. The last clean pellet was resuspended in $150 \mu \mathrm{l}$ of $200 \mathrm{mmol}^{-1}$ perchloric acid, shaken for $1 \mathrm{~min}$ and then centrifuged for $10 \mathrm{~min}$ at $2000 \mathrm{~g}$ at $4{ }^{\circ} \mathrm{C}$. The supernatant was then stored at $-80^{\circ} \mathrm{C}$ until used for noradrenaline estimation.

Blood was withdrawn from a second series of animals in extractions of $0.050 \mathrm{ml}$ (at $3 \mathrm{~min}$ intervals) and then deproteinized with perchloric acid (Merck); the acid was then removed with potassium bicarbonate and by centrifugation $(10000 \mathrm{~g}$ for $7 \mathrm{~min})$. The supernatants were stored at $-80^{\circ} \mathrm{C}$ until processed for glucose (Trinder, 1969) and 3-OHB (Williamson et al. 1962) estimation.

Noradrenaline was determined by using a high-performance liquid chromatography (HPLC) apparatus (model 1050, 
Hewlett-Packard, Palo Alto, CA, USA) fitted with an electrochemical detector (model 5100A, Coulochem, Bedford, MA, USA). Samples of $50 \mu 1$ of purified extracts and standards were injected into a $15 \mathrm{~cm} \times 0.46 \mathrm{~cm}$ column of Spherisorb ODS2 $5 \mu \mathrm{m}$ (Tecnokroma, Sant Cugat del Vallès, Spain), and a mobile phase of $100 \mathrm{mmoll}^{-1}$ sodium acetate, $40 \mathrm{mmoll}^{-1}$ citric acid, $60 \mathrm{mmol}^{-1}$ sodium hydroxide, $5 \mathrm{mmol}^{-1} 1$-octane sulphonic acid and $100 \mathrm{mll}^{-1}$ methanol, $\mathrm{pH} 5.2$, was run through at a pressure of $12.4 \mathrm{MPa}$ and a rate of $0.80 \mathrm{ml} \mathrm{min}^{-1}$ (Mefford et al. 1981). Noradrenaline was eluted as a distinct peak at $4.5-5 \mathrm{~min}$, adrenaline at $5.5-6 \mathrm{~min}$ and dihydroxybenzylamine at $8.5-9 \mathrm{~min}$. The assay was completed in $20 \mathrm{~min}$.

Estimation of differences between groups was performed using standard one- or two-way analysis of variance (ANOVA), and the Student's $t$-test was used for direct comparison between pairs of groups. The limit of significance was set at $P<0.05$.

\section{Results}

Plasma noradrenaline levels rose sharply at the beginning of the infusion and stabilized at approximately $50 \mathrm{nmoll}^{-1}$ (Fig. 1), a level that was maintained for up to $60 \mathrm{~min}$. The presence of 3-OHB in the infusion lowered the levels of noradrenaline significantly (to approximately $30 \mathrm{nmoll}^{-1}$ ). Removal of the 3-OHB infusion restored the noradrenaline levels.

Noradrenaline induced a gradual increase in aortic (core) temperature to $40-41{ }^{\circ} \mathrm{C}$. The addition of 3-OHB reduced the rate of temperture increase significantly (Fig. 2A). When 3OHB infusion was stopped, the rate of temperature increase was restored. Infusion of saline resulted in a slow decrease in core temperature; a similar slow decrease was obtained with glucose infusion. Infusion of 3-OHB alone (data not shown) also caused a similar pattern of temperature change. The replacement of 3-OHB by glucose in noradrenaline-infused rats had no effect on the rate of increase induced by noradrenaline alone (data not shown).

The temperature changes observed in the aorta reflected changes in the temperature of the interscapular BAT. Infusions of saline, glucose (Fig. 2B) or 3-OHB alone (data not shown) induced a slow decrease in temperature identical to that of aortic core temperature. Noradrenaline infusion resulted in an increase in interscapular BAT temperature to $41-42^{\circ} \mathrm{C}$. Infusion of 3-OHB and noradrenaline resulted in a marked slowing of the rate of increase in temperature, an effect that was maintained after the cessation of 3-OHB infusion. Substitution of 3-OHB by glucose (data not shown) resulted in the same pattern as described for noradrenaline alone. The differences between interscapular BAT and aortic temperature (i.e. the differential heating induced by BAT) in the rats infused with noradrenaline and 3-OHB are shown in Fig. 2C; a temperature difference of up to $2^{\circ} \mathrm{C}$ was maintained in noradrenaline-infused rats up to $45 \mathrm{~min}$ of infusion, after which this difference began to decrease at a comparable rate.

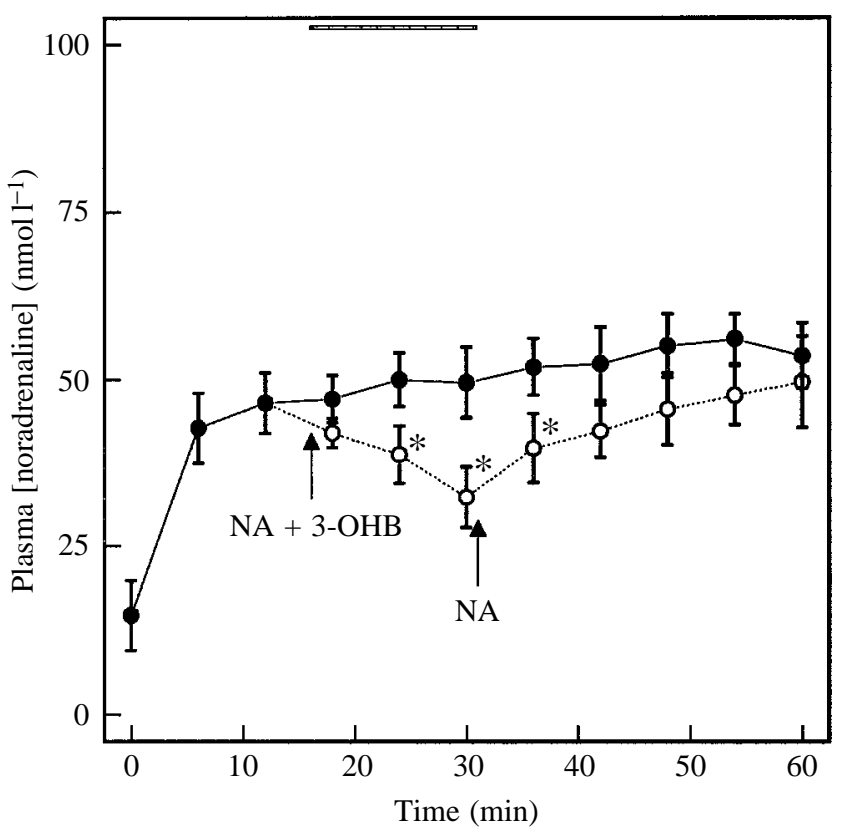

Fig. 1. Plasma noradrenaline levels in rats infused with noradrenaline (NA) (filled circles) or noradrenaline +3 -hydroxybutyrate (3-OHB) (open circles). The data represent the mean \pm S.E.M. for 7-8 different animals. The arrows indicate the period of infusion of 3-OHB (also marked as a bar at the top of the figure). Significance of the differences between groups (Student's $t$-test): the differences between the noradrenaline and noradrenaline +3 -OHB groups were significant only at 24,30 and $36 \mathrm{~min}$ (asterisks).

Moreover, 3-OHB infusion halted the rise in temperature differential, an effect that continued after infusion ceased.

3-Hydroxybutyrate levels were not affected by noradrenaline treatment. Infusion of 3-OHB increased noradrenaline concentration to above $1 \mathrm{mmoll}^{-1}$ (Fig. 3). After 3-OHB infusion ceased, levels fell again to the basal concentrations.

The curve for the decrease in blood 3-OHB levels after its infusion ceased was fitted to a decay equation:

$$
H=A e^{-K t},
$$

where $t$ is time, $H$ is the concentration of $3-\mathrm{OHB}, A$ is a constant, and $K$ is the decay constant. The half-time for this process was $4.8 \mathrm{~min}$.

Noradrenaline infusion increased blood glucose concentration gradually by almost $5 \mathrm{mmoll}^{-1}$ in $1 \mathrm{~h}$ (Fig. 4). However, infusion of 3-OHB reversed this effect, first causing a slow decrease in glucose concentration that then stabilized. The difference in blood glucose concentration compared with infusion of noradrenaline alone was almost $4 \mathrm{mmoll}^{-1}$.

\section{Discussion}

Anaesthesia affects thermoregulation in the rat; its effects are often associated with a fall in core temperature due to lower rate of heat production (Ohlson et al. 1992) and a diminished ability to retain heat, consistent with destabilized temperature 

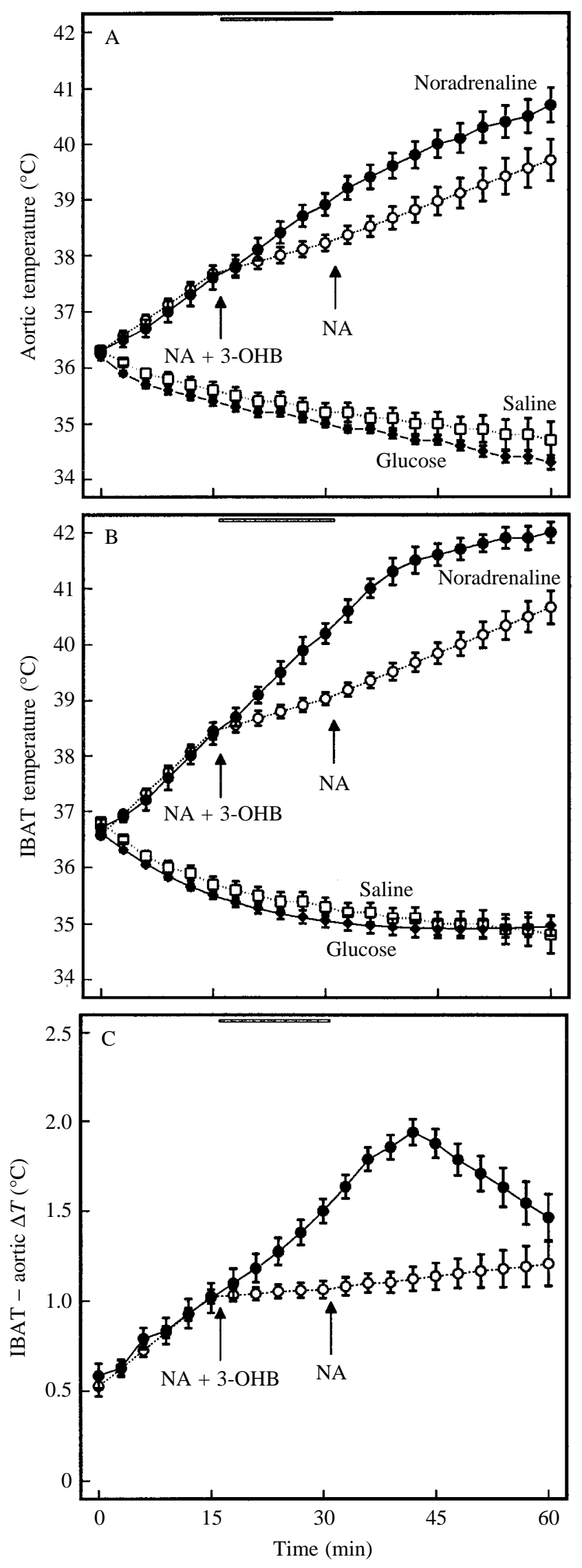

Fig. 2. Temperature changes in rats infused with noradrenaline (NA) (filled circles), noradrenaline $+3-\mathrm{OHB}$ (open circles), saline (open squares) or glucose (filled diamonds). (A) Aortic temperature; (B) interscapular brown adipose tissue (IBAT) temperature; (C) difference between IBAT and aortic temperatures $(\Delta T)$. Noradrenaline and noradrenaline +3 -OHB data represent the mean \pm S.E.M. for 9-10 different animals. Saline and glucose data are the mean \pm S.E.M. for 5-6 different animals. The arrows indicate the period of infusion of 3-OHB (also marked as a bar at the top of the figure). Significance of the differences between groups (ANOVA): (A) there were no differences between the saline- and glucoseinfused groups; the differences between the noradrenaline and noradrenaline +3 -OHB groups were significant, as were those between these two groups and the saline and glucose groups; (B) there were no significant differences between the saline- and glucose-infused groups; the differences between the noradrenaline and noradrenaline +3 -OHB groups were significant, as were those between them and the saline and glucose groups; $(\mathrm{C})$ the differences between both groups were significant.

adjustment mechanisms (Takemoto, 1992). The preparation used here cushions the fall in body temperature by maintaining a high environmental temperature. Infusion of saline or saline supplemented with 3-OHB or glucose elicited neither a rise in temperature nor an alteration in the basal hypothermic condition induced by pentobarbital anaesthesia (Sessler, 1991; Takemoto, 1992).

Most of the alterations to the effects of noradrenaline described here may be traced to the decrease in circulating

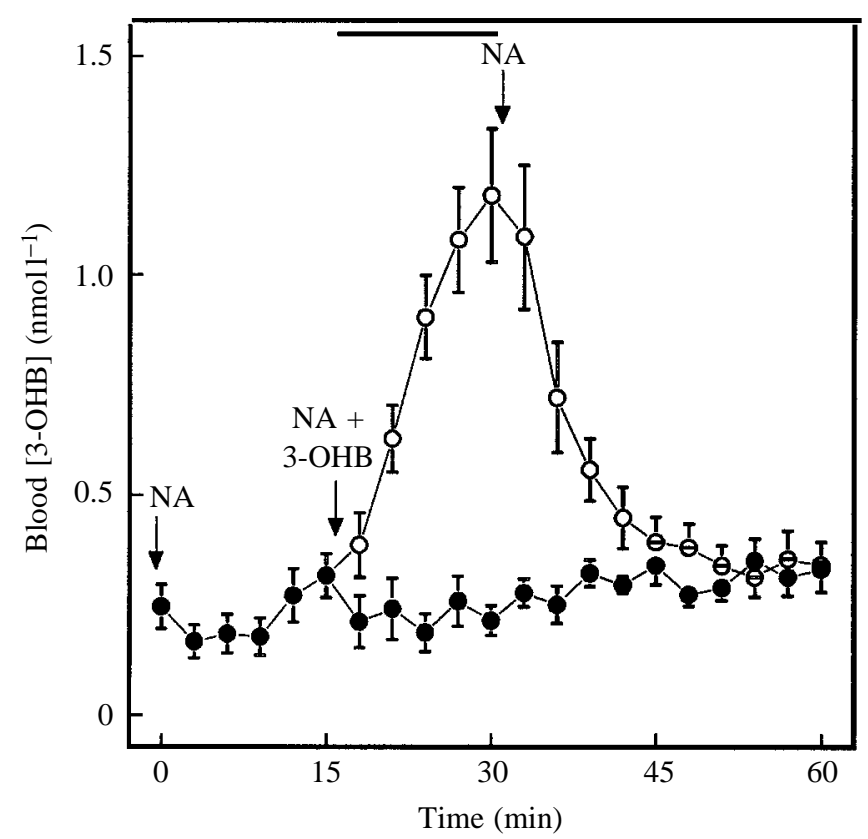

Fig. 3. Blood 3-OHB levels of rats infused with noradrenaline (NA) (filled circles) or noradrenaline $+3-\mathrm{OHB}$ (open circles). The data represent the mean \pm S.E.M. for $7-8$ different animals. Arrows indicate the period of infusion of 3-OHB (also marked as a bar at the top of the figure). Significance of the differences between groups (Student's $t$-test): the differences between the noradrenaline and noradrenaline + $3-\mathrm{OHB}$ groups were significant only between 22 and $40 \mathrm{~min}$. 


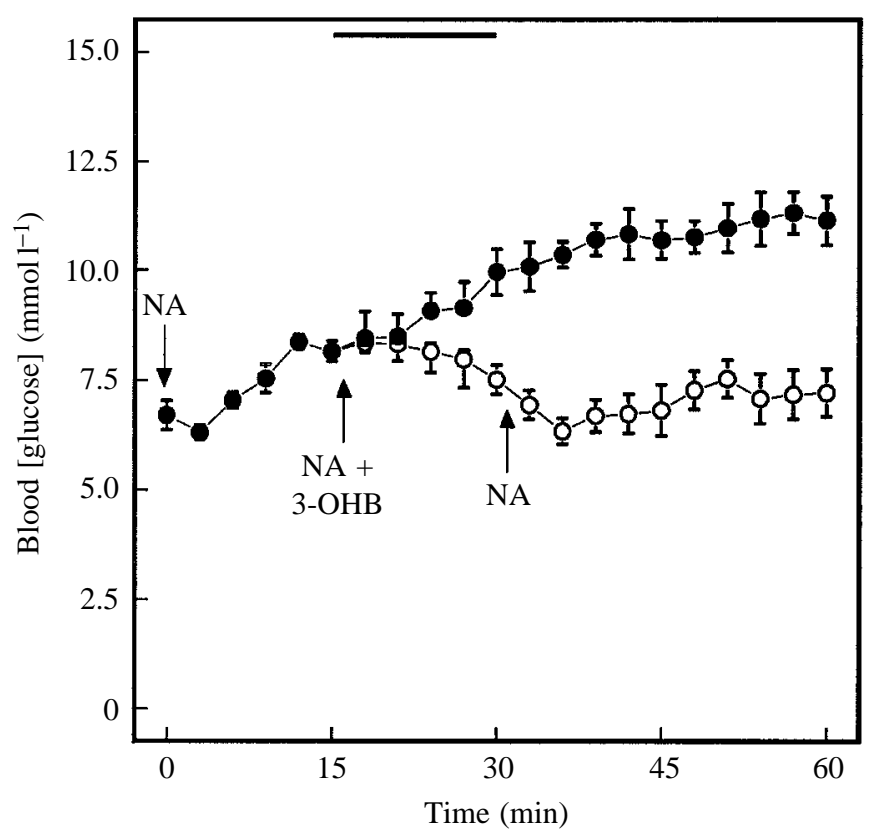

Fig. 4. Blood glucose levels of rats infused with noradrenaline (NA) (filled circles) or noradrenaline $+3-\mathrm{OHB}$ (open circles). The data represent the mean \pm S.E.M. for 7-8 different animals. Arrows indicate the period of infusion of 3-OHB (also marked as a bar at the top of the figure). Significance of the differences between groups (Student's $t$-test): the differences between the noradrenaline and noradrenaline + 3 -OHB groups were significant from 28 min onwards.

levels of noradrenaline induced by 3-OHB infusion. This transient effect cannot be reproduced by either saline or glucose infusion. However, it can explain, at least in part, the arrest by 3-OHB of temperature increases and the noradrenaline-induced rise in blood glucose level because, during 3-OHB infusion, there was less circulating noradrenaline left to act on BAT and the liver glycogenolytic pathway.

The observed effects of 3-OHB infusion on the adrenergic response may be directly related to its concentration in the plasma, since the maximal effect on BAT temperature coincides with the highest plasma 3-OHB level, a value close to the values reached during short-term starvation (Palou et al. 1981) but far below the upper physiological limit attainable during starvation (Balasse, 1979). The ability of the rat to remove 3-OHB from the blood was also concentrationdependent, suggesting that its utilization by peripheral tissues was enhanced by a fast turnover.

Noradrenaline elicits a significant thermogenic response in BAT, whose temperature differential from that of the aortic core was increased. Noradrenaline increases heat production in BAT (Foster and Frydman, 1978) and other tissues (Ma et al. 1987), thus raising core temperature (Foster and Frydman, 1978), as observed here, by promoting heat storage. The infusion of 3-OHB strongly influences the effect of noradrenaline on BAT temperature, i.e. on BAT heat production. This agrees with its role as a modulator of adrenergically induced thermogenesis when energy substrates are scarce, such as during starvation. The effect of 3-OHB on the stimulation of brown adipocytes by noradrenaline (Pokasanieva et al. 1993) may be related to its direct effects on $\beta$-adrenergic receptors (De Pergola et al. 1990; Gómez et al. 1996), but the overall effect is a 3-OHB-induced decrease in BAT thermogenesis.

Glucose levels increased during noradrenaline infusion, a direct consequence of generalized glycogenolysis (Shiota et al. 1992). The increase in temperature observed may result in higher demands for substrates, e.g. glucose, to fuel the increased thermogenesis, since this is the main substrate for the BAT thermogenesis that is elicited by noradrenaline or exposure to cold (Foster and Frydman, 1978, 1979). The slow rise in blood glucose levels reflects the balance between noradrenaline-elicited release of glucose from glycogen and its consumption by BAT and other tissues, which is also in part dependent on noradrenaline stimulation. The rapid utilization of infused 3-OHB as an alternative source of energy would spare glucose and tend to increase glucose levels (Senior and Loridan, 1968; Amiel et al. 1991). In spite of this sparing of glucose stores, plasma glucose levels actually decreased in response to 3-OHB infusion, which suggests that its effects on glycogenolysis are even more marked than those on glucose consumption. Thus, it may be assumed that 3-OHB inhibits noradrenaline-induced glycogenolysis by decreasing the levels of circulating noradrenaline but also by altering the response of adrenergic receptors to this hormone (De Pergola et al. 1990; Gómez et al. 1996).

However, the decrease in circulating noradrenaline levels elicited by 3-OHB is difficult to explain, since the rate of infusion of noradrenaline did not change throughout the experiment: a decrease in noradrenaline levels could only be explained by an increase in its rate of removal/destruction. This may be due to enhanced disposal of the hormone, which in this case would be dependent on the concentration of 3-OHB. The decreased affinity of $\beta$-adrenergic receptors for noradrenaline (De Pergola et al. 1990; Gómez et al. 1996) and the modulation of central sympathetic brain activity induced by 3-OHB (Maekubo et al. 1977; Fisler et al. 1995) may contribute to the role of 3-OHB as an important regulating agent that shows multiple interactions with noradrenaline within the physiological concentration range. This interaction may be part of an elaborate and complex fail-safe mechanism to prevent wastage of stored substrates.

Thanks are given to Robin Rycroft for correction of the text. The authors wish to thank the personnel of the Animal Service of Division III of the University of Barcelona for help and care of the animals. This work was supported in part by the 'Fondo de Investigaciones Sanitarias' (projects 94/34-01 and 95/266) of the Government of Spain.

\section{References}

Amiel, S. A., Archibald, H. R., Chusney, G., Williams, A. J. K. 
AND GAle, E. A. M. (1991). Ketone infusion lowers hormonal responses to hypoglycaemia: evidence for acute cerebral utilization of a non-glucose fuel. Clin. Sci. 81, 189-194.

BaLASSE, E. O. (1979). Kinetics of ketone body metabolism in fasting humans. Metabolism 28, 41-50.

Balasse, E. O. AND FÉRY, F. (1989). Ketone body production and disposal: effects of fasting, diabetes and exercise. Diabetes Metabol. Rev. 5, 247-270.

Closa, D., Gómez-Sierra, J. M., Latres, E., Alemany, M. And REMESAR, X. (1993). Short-term oscillations of aortic core temperature and thermogenic organ blood-flow in the rat. Exp. Physiol. 78, 243-253.

De Pergola, G., Cignarelli, M., Corso, M., Garruti, G., Di Paolo, S. AND GIORgINO, R. (1990). D-(-) $\beta$-hydroxybutyrate inhibits catecholamine-stimulated lipolysis and decreases $\beta$ adrenoreceptors' affinity in human fat cells but not in lymphomonocytes. Acta endocr. 122, 450-454.

FÉRY, F. AND BAlASSE, E. O. (1985). Ketone body production and disposal in diabetic ketosis. A comparison with fasting ketosis. Diabetes 34, 326-332.

Fisler, J. S., Egawa, M. And Bray, G. A. (1995). Peripheral 3hydroxybutyrate and food intake in a model of dietary-fat induced obesity: Effect of vagotomy. Physiol. Behav. 1, 1-7.

Foster, D. O. AND Frydman, M. L. (1978). Nonshivering thermogenesis in the rat. II. Measurements of blood flow with microspheres point to brown adipose tissue as the dominant site of the calorigenesis induced by noradrenaline. Can. J. Physiol. Pharmac. 56, 110-122.

Foster, D. O. And Frydman, M. L. (1979). Tissue distribution of cold-induced thermogenesis in conscious warm- or cold-acclimated rats reevaluated from changes in tissue blood flow: the dominant role of brown adipose tissue in the replacement of shivering by nonshivering thermogenesis. Can. J. Physiol. Pharmac. 57, 257-270.

Gómez, G., Cañas, X., Fernández-López, J. A., Remesar, X. and AlEMANY, M. (1976). 3-Hydroxybutyrate decreases noradrenaline affinity for rat erythrocyte ghost $\beta_{2}$-adrenergic receptors. Biochem. molec. Biol. Int. 40, 373-378.

Gordon, C. J. (1990). Thermal biology of the laboratory rat. Physiol. Behav. 47, 963-991.

Hetenyi, G. J. and Ferrarotto, C. (1985). Gluconeogenesis from acetone in starved rats. Biochem. J. 231, 151-155.

Hughes, I. E. And SMith, J. A. (1978). The stability of noradrenaline in physiological saline solutions. J. Pharm. Pharmac. 30, 124-126.

Hugie, T., Halvorson, I. AND Thornhill, J. (1992). Brown adipose tissue temperature responses following electrical stimulation of ventromedial hypothalamic and lateral preoptic areas or after norepinephrine infusion to Long Evans or Sprague-Dawley rats. Brain Res. 575, 57-62.

Ide, T., Yamamoto, I. And Sugano, M. (1984). Effect of dietary fat on lipid secretion and ketone body production in rat liver. J. Nutr. Sci. Vitaminol. 30, 153-162.

Ma, S. W., NAdeau, B. E. AND Foster, D. O. (1987). Evidence for the liver as the major site of diet-induced thermogenesis of rats fed a 'cafeteria' diet. Can. J. Physiol. 65, 1802-1804.

Maekubo, H., Moriya, K. And Hiroshige, T. (1977). Role of ketone bodies in nonshivering thermogenesis in cold-acclimated rats. $J$. appl. Physiol. 42, 159-165.

Mefford, I. N., Ward, M. M., Miles, L., Taylor, B., Chesney, M. A., Keegan, D. L. AND Barchas, J. D. (1981). Determination of plasma catecholamines and free 3,4-dihydroxyphenylacetic acid in continuously collected human plasma by high performance liquid chromatography with electrochemical detection. Life Sci. 28, 477-483.

Metcalfe, H. K., Monson, J. P., DeAllie, F. and Cohen, R. D. (1992). Effects of D-3-hydroxybutyrate and acetoacetate on lactate removal in isolated perfused livers from starved and fed rats. Metabolism 41, 435-440.

Nikami, H., Shimizu, Y., Endoh, D., Yano, H. And SAIto, M. (1992). Cold-exposure increases glucose-utilization and glucose transporter expression in brown adipose-tissue. Biochem. biophys. Res. Commun. 3, 1078-1082.

Ohlson, K., Dicker, A., Mohell, N., Lindahl, S., CANnON, B. AND NeDERGAARD, J. (1992). A specific inhibitory effect of halothane on brown adipose tissue thermogenesis. In Thermoregulation: The Pathophysiological Basis of Clinical Disorders (ed. P. Lomax and E. Schönbaum), pp. 42-45. Basel: Karger.

OHMORI, H., KawaI, K. And Yamashita, K. (1990). Enhanced ketone body uptake by perfused skeletal muscle in trained rats. Endocr. Japon. 37, 421-429.

Palou, A., Remesar, X., Arola, L., Herrera, E. and Alemany, M. (1981). Metabolic effects of short term food deprivation in the rat. Horm. Metabol. Res. 13, 326-330.

Pokasanieva, E. B., Cañas, X., Closa, D., Remesar, X. and AlEMANY, M. (1993). 3-Hydroxybutyric acid arrests brown adipose tissue thermogenesis elicited by noradrenaline. In Obesity in Europe (ed. H. Ditschuneit, F. A. Gries, H. Hauner, V. Schusdziarra and J. G. Wechsler), pp. 127-134. London: John Libbey.

Rothwell, N. J. AND STOCK, M. J. (1979). A role for brown adipose tissue in diet-induced thermogenesis. Nature 281, 31-35.

SENIOR, B. AND LORIDAN, L. (1968). Direct regulatory effect of ketones on lipolysis and on glucose concentrations in man. Nature 219, 83-84.

SESSLER, D. I. (1991). Central thermoregulatory inhibition by generalanesthesia. Anesthesiology 4, 557-559.

Sherwin, R. S., Hendler, R. G. AND Felig, P. (1976). Effect of diabetes mellitus and insulin on the turnover and metabolic response to ketones in man. Diabetes 25, 776-784.

Shiota, M., Kurano, Y., Mochizuki, Y., Kimura, K., OHTA, M. AND SugAnO, T. (1992). Effects of nerve-stimulation and zymosan on glycogenolysis in perfused livers from cold-exposed rats. Am. J. Physiol. 263, G353-G359.

TAкEмото, Y. (1992). Decreases in catecholamine concentrations of cisternal cerebrospinal fluid and plasma in rats caused by pentobarbital anesthesia. Jap. J. Physiol. 42, 141-145.

Trayhurn, P. AND Ashwell, M. (1987). Control of white and brown adipose tissues by the autonomic nervous-system. Proc. Nutr. Soc. 46, 135-142.

Trinder, P. (1969). Determination of glucose in blood using glucose oxidase with an alternative oxygen acceptor. Annls clin. Biochem. 6, 557-559.

Ubukata, E., Mokuda, O., SaKamoto, Y. and Shimizu, N. (1992). Effect of FFA on ketone production in rat's liver perfusion system. Horm. Metabol. Res. 24, 134-135.

WEST, G. B. (1952). The stability of noradrenaline solutions. $J$. Pharm. Pharmac. 4, 550-565.

Williamson, D. H., Mellanby, J. And Krebs, H. A. (1962). Enzymic determination of $\mathrm{D}(-)-\beta$-hydroxybutyric acid and acetoacetic acid in blood. Biochem. J. 82, 90-96.

Zylan, K. D. AND Carlisle, H. J. (1992). Effect of ambient temperature on the paradoxical metabolic responses to norepinephrine. Pharmacol. Biochem. Behav. 43, 577-582. 